\title{
APPROXIMATION OF PLURISUBHARMONIC FUNCTIONS ON COMPLEX VARIETIES
}

\author{
NGUYEN QUANG DIEU, TANG VAN LONG AND SANPHET OUNHEUAN
}

\section{INTRODUCTION}

Let $V$ be a complex variety in a domain $D \subset \mathbb{C}^{n}(n \geq 2)$ i.e, $V$ is a closed subset of $D$ and for every $z_{0} \in V$, there exists a neighborhood $U$ of $z_{0}$ such that $U \cap V$ is the common zero set of holomorphic functions on $U$. Let $P S H(V)$ denote the cone of plurisubharmonic functions on $V$. Recall that a function $u: V \rightarrow[-\infty, \infty)$ is plurisubharmonic if $u$ is locally the restriction (on $V$ ) of a plurisubharmonic functions on an open subset of $D$. Notice that we regard the function identically $-\infty$ as plurisubharmonic. A fundamental result of Fornaess and Narasimhan (cf. Theorem 5.3.1 in [FN]) asserts that an upper semicontinuous function $u: V \rightarrow \mathbb{R} \cup[-\infty, \infty)$ is plurisubharmonic if and only if for every holomorphic map $\theta: \Delta \rightarrow V$, where $\Delta$ is the unit disk in $\mathbb{C}$, we have $u \circ \theta$ is subharmonic on $\Delta$. This powerful result implies immediately the nontrivial facts that plurisubharmonicity is preserved under uniform convergence.

We write $P S H(V)$ for the set of plurisubharmonic functions on $V$. In this paper, we address the question of approximating elements of $P S H(V)$ which are bounded from above by plurisubharmonic functions on $V$ which are continuous on $V$ or on $\bar{V}$. After subtracting constants, we need only consider functions in $\mathrm{PSH}^{-}(V)$ the convex cone of plurisubharmonic functions $u$ on $V$ such that the upper semicontinuous regularization

$$
u^{*}(\xi):=\varlimsup_{z \rightarrow \xi, z \in V} u(z)<0, \forall \xi \in \partial V .
$$

Our main tool is is a duality theorem of Edwards which expresses upper envelopes of plurisubharmonic functions taken in convex sub-cones of $\mathrm{PSH}(V)$ in terms of Jensen measures with respect to these cones. This approach has been used [Wik], [DW] and [Di] in the case where $V$ is a bounded domain in $\mathbb{C}^{n}$. The general principle is that the approximation of elements in a cone $\mathscr{A} \subset P S H^{-}(V)$ by elements in a smaller cone $\mathscr{B}$ is possible when we have equality of the set of Jensen measures with respect to $\mathscr{A}$ and $\mathscr{B}$. Nevertheless, in our setting, there are at least two difficulties, first the standard local smoothing by convolving with approximate identities is not possible on the complex variety $V$, the second one stems from the fact that (the upper semicontinuous regularization of) the supremum of a family of plurisubharmonic functions which are locally uniformly bounded from above is not necessarily plurisubharmonic (cf. Example 1.4 in [Ze]). Therefore, we have to make extra conditions to overcome these obstacles. Namely, for the first one, the variety $V$ is assumed to be Stein i.e., there exists a plurisubharmonic exhaustion function on $V$, so that a global approximation theorem of Fornaess and Narasimhan (cf. Theorem 5.5 in [FN]) is applicable (cf. Theorem 1.4), the other one is settled by putting some restriction on the part of $V$ which fails to be locally irreducible (cf. Theorem 1.3).

In order to formulate our results properly, it is convenient to introduce the following notions pertaining to our work.

Date: October 15, 2018.

2000 Mathematics Subject Classification. Primary 32U15; Secondary 32B15. 
Definition 1.1. Let $V$ be a complex variety in a domain $D \subset \mathbb{C}^{n}$. For a point $z \in \bar{V}$, we define two classes of Jensen measures

$$
\begin{aligned}
& J_{z}=\left\{\mu \in \mathscr{B}(\bar{V}): u(z) \leq \int_{\bar{V}} u d \mu, \forall u \in \operatorname{PSH}^{-}(V)\right\} \\
& J_{z}^{c}=\left\{\mu \in \mathscr{B}(\bar{V}): u(z) \leq \int_{\bar{V}} u d \mu, \forall u \in \operatorname{PSH}_{c}^{-}(V)\right\}
\end{aligned}
$$

where $\mathrm{PSH}_{c}^{-}(V)$ is the cone of negative continuous functions on $\bar{V}$ which is plurisubharmonic on $V$ and $\mathscr{B}(\bar{V})$ denotes the set of Borel probability measures with support on $\bar{V}$.

The connection between Jensen measures and approximation of plurisubharmonic functions stems from the following fact which is a simple consequence of Fatou's lemma.

Proposition 1.2. Let $E$ be a subset of $V$ such that for every $u \in P S H^{-}(V)$, there exists a sequence $\left\{u_{j}\right\}_{j \geq 1} \subset \mathrm{PSH}_{c}^{-}(V)$ having the following properties:

(i) $u_{j} \rightarrow u$ pointwise on $E$.

(ii) $\varlimsup_{j \rightarrow \infty} v_{j} \leq u$ on $V$.

Then $J_{z}=J_{z}^{c}$ for every $z \in E$.

In the opposite direction, the next result gives a sufficient condition so that pointwise approximation of negative plurisubharmonic functions on complex varieties by continuous plurisubharmonic ones is possible. Before formulating it, recall that a complex variety $V \subset D \subset \mathbb{C}^{n}$ is said to be locally irreducible if it is so at at every point $a \in V$ i.e., there is a fundamental system of neighborhoods $U_{j}$ of $a$ such that $U_{j} \cap V$ is irreducible in $U_{j}$ for every $j$. This point of local irreducibility was recorded incorrectly in Definition 1.1 of [Wik2] where it only requires a single neighborhood $U$ of $a$ such that $U \cap V$ is locally irreducible. In general, it is quite hard to decide whether $V$ is locally irreducible near its singular locus. Under some restriction on the set of local irreducible points of $V$ we have the following result.

Theorem 1.3. Let $V$ be a complex variety in a bounded domain $D \subset \mathbb{C}^{n}$. Assume that there exists $\psi \in \mathrm{PSH}^{-}(V), \psi \not \equiv-\infty$ satisfying the following conditions:

(i) $F:=\{z \in V: \psi(z)=-\infty\}$ is a closed subset of $V$.

(ii) $V \backslash F$ is a locally irreducible complex variety in $D \backslash F$.

Suppose that there exists $E \subset V$ such that $J_{z}=J_{z}^{c}$ for all $z \in V \backslash E$. Then for every $u \in$ $\mathrm{PSH}^{-}(V), u^{*}<0$, there exists two sequences $\left\{u_{j}\right\}_{j \geq 1} \subset \mathrm{PSH}^{-}(V \backslash F)$ and $\left\{v_{j}\right\}_{j \geq 1} \subset \mathrm{PSH}_{c}^{-}(V)$ having the following properties:

(a) $u_{j} \downarrow u$ on $V \backslash F$ and $u_{j}$ is continuous at every point of $V \backslash(E \cup F)$.

(b) $v_{j} \rightarrow u$ pointwise on $V \backslash(\bar{E} \cup F)$ and $\varlimsup_{j \rightarrow \infty} v_{j} \leq u^{*}$ on $\bar{V}$.

(c) Suppose in addition that $V$ is of pure dimension $k, \bar{E}$ is pluripolar in $V$ and $u$ is locally bounded on $V$. Then the sequence $\left\{v_{j}\right\}_{j \geq 1}$ can be chosen to be locally uniformly bounded on $V$ and $\left(d d^{c} v_{j}\right)^{k} \rightarrow\left(d d^{c} u\right)^{k}$ on $U$ in the weak ${ }^{*}$-topology as $j \rightarrow \infty$.

We prefer to postpone a brief discussion on pluripolar sets and Monge-Ampère operator on the complex variety $V$ in the next section. Several remarks concerning Theorem 1.3 are now in order. 
Remarks. (a) We do not know if there always exists a function $\psi$ that satisfies (i) and (ii) even in the case where $V$ is Stein. However, if $V$ is a complex variety in a bounded pseudoconvex domain $D^{\prime}$ that contains $\bar{D}$ then such a function $\psi$ can be found as follows. Since the singular part of $V$, denoted by $V_{s}$ is a (proper) complex subvariety of $V$, we deduce that $V_{s}$ is also a complex subvariety of $D^{\prime}$. Thus, $V_{s}=\left\{z \in D^{\prime}: f_{1}(z)=\cdots=f_{k}(z)=0\right\}$, where $f_{1}, \cdots, f_{k}$ are holomorphic functions on $D^{\prime}$. It is clear that $\psi:=\log \left(\left|f_{1}\right|+\cdots+\left|f_{k}\right|\right)-M \in \operatorname{PSH}^{-}(V)$ is the desired plurisubharmonic function for $M>0$ large enough.

(b) We should say that in the case where $V$ is an open subset of $\mathbb{C}^{n}$ and $E=\emptyset$, the assertions (a) and (b) are implicitly contained in Theorem 3.1 of [DW]. We thought it may be of interest to include the case where there exists some exceptional set $E$ so that the two classes of Jensen measures may differ on $E$.

(c) The statement (c) was essentially proved in Theorem of [Di]. Nevertheless, the somewhat complicated proof given there does not even generalize to the case $V$ is a smooth complex variety since it uses convolutions with smoothing kernels.

The next main result deals with the case where the exceptional set $E$ mentioned in Theorem might occurs.

Theorem 1.4. Let $V$ be a Stein complex variety in a bounded domain $D \subset \mathbb{C}^{n}$. Suppose that there exists $v \in P_{S H}^{-}(V), v \not \equiv-\infty$ and a compact $K \subset \partial V$ satisfying the following properties:

(i) $\lim _{z \rightarrow \xi} v(z)=-\infty, \forall \xi \in K$;

(ii) $J_{\xi}^{c}=\left\{\delta_{\xi}\right\}, \forall \xi \in(\partial V) \backslash K$.

Then the following statements hold true:

(a) For every $z \in V \backslash E$, where $E:=\{z \in V: v(z)=-\infty\}$ we have $J_{z}=J_{z}^{c}$.

(b) Suppose in addition that $V$ is locally irreducible, then for every $u \in \mathrm{PSH}^{-}(V)$ there exists a sequence $u_{j} \in \mathrm{PSH}^{-}(V)$ such that $u_{j}$ is continuous at every point of $U:=\bar{V} \backslash(K \cup E)$ and $u_{j}^{*} \downarrow u^{*}$ on $U$.

Remarks. (a) The condition (ii) is fulfilled at $\xi \in \partial V$ if there is a local continuous plurisubharmonic barrier at $\xi$ i.e., there exists $u \in \operatorname{PSH}_{c}^{-}(V \cap \mathbb{B}(\xi, r))$ for some small $\mathbb{B}(\xi, r) \subset \mathbb{C}^{n}$ such that $u(\xi)=0$ whereas $u<0$ elsewhere. Indeed, by shrinking $r$ we may assume that $u<-\delta$ for $\bar{V} \cap \partial \mathbb{B}(\xi, r)$ for some $\delta>0$. By setting $\tilde{u}:=\max \{u,-\delta\}$ on $\bar{V} \cap \mathbb{B}(\xi, r)$ and $\tilde{u}:=-\delta$ out of $\mathbb{B}(\xi, r)$ and using the gluing lemma (cf. Lemma 2.3) we have $\tilde{u} \in P S H_{c}^{-}(V)$ and satisfies $\tilde{u}(\xi)=0, \tilde{u}<0$ elsewhere. It easily implies that $J_{\xi}=\left\{\delta_{\xi}\right\}$. This reasoning is essentially contained in Proposition 1.4 in [Si].

(b) Let $\varphi(z, w):=|z|^{4}+|w|^{4},(z, w) \in \mathbb{C}^{2}$ and $\mathbb{B}_{2}$ be the unit ball in $\mathbb{C}^{2}$. We set

$$
D:=\left\{(z, w, t) \in \mathbb{B}_{2} \times \mathbb{C}:|t|<e^{-\varphi(z, w)}\right\}, V:=\left\{(z, w, t) \in D: t^{2}=z^{2} w\right\} .
$$

Then $V$ is a complex variety in the (bounded) Hartogs pseudoconvex domain $D$. Notice that, $V$ is locally reducible at every point $(0, w, 0) \in D$ with $0<|w|<1$ (cf. [Ch] p. 56). Given $\xi_{0}=$ $\left(z_{0}, w_{0}, t_{0}\right) \in \partial V, z_{0} w_{0} \neq 0$. By strict plurisubharmonicity of $\varphi$ at $\left(z_{0}, w_{0}\right)$ and strict pseudoconvexity of $\partial \mathbb{B}_{2}$ we see that every Jensen measure $\mu \in J_{\xi_{0}}$ is supported at $\left\{\xi_{0}\right\} \cup\left\{\left(z_{0}, w_{0},-t_{0}\right)\right\}$. It follows that $J_{\xi_{0}}=\left\{\delta_{\xi_{0}}\right\}$. Thus we may apply Theorem 1.4 with

$$
K:=\{(z, 0,0):|z|=1\} \cup\{(0, w, 0):|w|=1\}, v(z, w):=\log |z w|
$$

and get $E=\emptyset$. The above construction is also inspired from an example of Sibony (cf. [Si], p. $310)$. 
The theorem below deals with the problem of finding a bounded continuous maximal plurisubharmonic $u$ on $V$ such that the boundary values of $u$ coincides with a given continuous function defined on part of the boundary $\partial V$. A weaker version of this result is given in Theorem 1.8 of [Wik2] under the assumption that $V$ admits a $B$-regular neighborhood in $\mathbb{C}^{n}$. Recall that $u \in P S H(V)$ is said to be maximal if for every relatively compact open subset $U$ of $V$ and every $v \in P S H(V)$ such that $v \leq u$ on $V \backslash U$ we have $v \leq u$ on $V$. This definition is taken from Definition 1.6 in [Wik2] and is analogous to the classical one given by Sadullaev for the case where $V$ is an open set of $\mathbb{C}^{n}$ (cf. Proposition 3.1.1 in [K1]).

Theorem 1.5. Let $V$ be a Stein locally irreducible complex variety in a bounded domain $D \subset$ $\mathbb{C}^{n}$. Suppose that there is $v \in P H^{-}(V), v>-\infty$ on $V$ and a compact $K \subset \partial V$ satisfying the following properties:

(i) $\lim _{z \rightarrow \xi} v(z)=-\infty, \forall \xi \in K$;

(ii) Every $\xi \in(\partial V) \backslash K$ admits a local continuous plurisubharmonic barrier.

Then for every $\varphi \in \mathscr{C}(\partial V)$, there exists a unique bounded, maximal continuous plurisubharmonic function $u$ on $V$ such that

$$
\lim _{z \rightarrow \xi, z \in V} u(z)=\varphi(\xi), \forall \xi \in(\partial V) \backslash K
$$

Our final result generalizes Theorem 2.3 in [Wik2] in that it does not assume the existence of a $B$-regular neighborhood of $V$ in $\mathbb{C}^{n}$.

Theorem 1.6. Let $V$ be a complex variety in a bounded domain $D \subset \mathbb{C}^{n}$ having the following properties:

(i) $J_{\xi}^{c}=\left\{\delta_{\xi}\right\}, \forall \xi \in \partial V$.

(ii) There exists a negative continuous plurisubharmonic exhaustion function $\rho$ for $V$.

Then for every $u \in \mathrm{PSH}^{-}(V)$ there exists a decreasing sequence $\left\{u_{j}\right\}_{j \geq 1} \subset \mathrm{PSH}_{c}^{-}(V)$ such that $u_{j} \downarrow u^{*}$ on $\bar{V}$.

Remarks. (a) According to Theorem 1.4(b), under the additional assumption that $V$ is Stein locally irreducible the conclusion of the theorem is still valid without assuming (ii).

(b) The function $-1 / \rho(z)+|z|^{2}$ is continuous strictly plurisubharmonic on $V$ and tends to $+\infty$ as $z \rightarrow \partial V$. In particular, $V$ is Stein.

(c) If $V$ satisfies $(i i)$ alone then every $\mu \in J_{\xi}^{c}$ is supported on $\partial V$. This follows easily from the estimate $0=\rho(\xi) \leq \int_{\bar{V}} \rho d \mu=\int_{V} \rho d \mu$.

\section{PRELiminaries}

We recall the general version of Edward's duality theorem which says that upper envelopes of upper semicontinuous functions defined on compact metric spaces may be expressed as lower envelopes of integrals with respect to certain classes of measures.

Let $X$ be a compact metric space, and let $\mathscr{F}$ be a convex cone of real-valued, bounded from above and upper semicontinuous functions on $X$ containing all the constants. If $g$ is a realvalued function on $X$, then we define

$$
\operatorname{Sg}(z):=\sup _{4}\{u(z): u \in \mathscr{F}, u \leq g\} .
$$


Denote by $\mathscr{B}(X)$ the class of positive, regular Borel measures on $X$. For $z \in X$ we define

$$
J_{z}^{\mathscr{F}}:=\left\{\mu \in \mathscr{B}(X): u(z) \leq \int_{X} u d \mu, \forall u \in \mathscr{F}\right\} .
$$

It is easy to see that $J_{z}^{\mathscr{F}}$ is a convex, weak-* compact subset of $\mathbb{B}(X)$. Moreover, $\mu(X)=1$ for every $\mu \in J_{z}^{\mathscr{F}}$ since $\mathscr{F}$ contains the constants. On the other hand, if $\mathrm{g}$ is a Borel measurable function on $\mathrm{X}$, then we set

$$
\operatorname{Ig}(z):=\inf \left\{\int_{X} g d \mu: \mu \in J_{z}^{\mathscr{F}}\right\} .
$$

Now we are able to formulate the following basic duality theorem of Edwards (cf. [Ed], [Wik1]).

Theorem 2.1. Let $\mathscr{F}$ be as above. If $g$ is lower semicontinuous on $X$, then $S g=I g$.

Apparently the first use of Edwards's duality theorem in pluripotential theory has been made in the seminal work [Si] where we can find a systematic study of domains in $\mathbb{C}^{n}$ on which the Dirichlet problem for plurisubharmonic functions is solvable.

In our context, by applying the above theorem to the convex cones $\mathscr{F}_{1}:=\operatorname{PSH}^{-}(V)$ and $\mathscr{F}_{2}:=P S H_{c}^{-}(V)$ we obtain the following result which will be refereed to as Edward's duality theorem.

Theorem 2.2. (Edward's theorem) Let $\varphi: \bar{V} \rightarrow(-\infty,+\infty]$ be a lower semicontinuous function. Then we have

$$
\begin{aligned}
& \inf \left\{\int_{\bar{V}} \varphi d \mu, \mu \in J_{z}\right\}=\sup \left\{u(z): u \in P S H^{-}(V), u^{*} \leq \varphi \text { on } \bar{V}\right\}, \\
& \inf \left\{\int_{\bar{V}} \varphi d \mu, \mu \in J_{z}^{c}\right\}=\sup \left\{u(z): u \in \operatorname{PSH}_{c}^{-}(V), u \leq \varphi \text { on } \bar{V}\right\} .
\end{aligned}
$$

We will make a good use of the following result about gluing plurisubharmonic functions on complex varieties. This fact has been also used implicitly in the proof of Theorem 1.8 of [Wik2] .

Lemma 2.3. Let $V$ be a complex variety of a domain $D \subset \mathbb{C}^{n}, U \subset V$ be an open subset and $u \in P S H(V), v \in P S H(U)$. Assume that $\varlimsup_{z \rightarrow \xi} v(\xi) \leq u(z) \forall z \in \partial U$. Then the function

$$
w:= \begin{cases}\max \{u, v\} & \text { on } U \\ u & \text { on } V \backslash U .\end{cases}
$$

belongs to $\mathrm{PSH}(V)$.

Proof. The proof uses again the above mentioned Fornaess-Narasimhan's criterion for membership in $P S H(V)$. More precisely, by the assumption $w$ is upper semicontinuous on $V$, so it remains to check that $w \circ \theta$ is subharmonic on $\Delta$ for every holomorphic map $\theta: \Delta \rightarrow V$. Clearly

$$
w \circ \theta= \begin{cases}\max \{u \circ \theta, v \circ \theta\} & \text { on } \theta^{-1}(U) \\ u \circ \theta & \text { on } \Delta \backslash \theta^{-1}(U) .\end{cases}
$$

Since $\theta^{-1}(U)$ is an open subset of $\Delta$ we see that if $\lambda \in \partial\left(\theta^{-1}(U)\right)$ then $\theta(\lambda) \in \partial U$, and hence

$$
\limsup _{\theta^{-1}(U) \ni t \rightarrow \lambda} v \circ \theta(t) \leq \limsup _{U \ni z \rightarrow \theta(\lambda)} v(z) \leq u \circ \theta(\lambda)
$$


Thus we may apply the usual gluing lemma for subharmonic functions to reach that $w \circ \theta$ is subharmonic on $\Delta$. The proof is complete.

The following fact about plurisubharmonicity of upper envelopes of plurisubharmonic functions on complex varieties which may not be locally irreducible is needed in the proof of Theorem 1.3.

Lemma 2.4. Let $V$ be a complex variety of a bounded domain $D \subset \mathbb{C}^{n}$. Assume that there exists $\psi \in \mathrm{PSH}^{-}(V), \psi \not \equiv-\infty$ satisfying the conditions (i) and (ii) in Theorem 1.3 Then for every upper semicontinuous function $\varphi: V \rightarrow[-\infty, 0)$ we have $v=v^{*} \in \operatorname{PSH}^{-}(V \backslash F)$, where

$$
v(z):=\sup \left\{u(z): u \in P_{S H}^{-}(V), u \leq \varphi \text { on } V\right\}, z \in V .
$$

Proof. First, by the assumption on $\varphi$ we have $v<0$ on $V$. This implies that $v^{*}$ is negative and plurisubharmonic on the regular part of $V$. In view of (ii), we may apply the theorem on removable singularities for plurisubharmonic functions (cf. Theorem 1.7 in [De]) to conclude that $v^{*} \in P S H^{-}(V \backslash F)$. Moreover, since $\varphi$ is upper semicontinuous, $v^{*} \leq \varphi$ on $V$.

Next, for $\varepsilon>0$ we set

$$
v_{\varepsilon}:= \begin{cases}v^{*}+\varepsilon \psi & \text { on } V \backslash F \\ -\infty & \text { on } F .\end{cases}
$$

Then $v_{\varepsilon} \leq \varphi<0$ and upper semicontinuous on $V$. Moreover, by (i), for any holomorphic mapping $\theta: \Delta \rightarrow V$, the composition map $v_{\varepsilon} \circ \theta$ is subharmonic on the open set $\Delta \backslash \theta^{-1}(F)$ and equal to $-\infty$ on $\theta^{-1}(F)$. It follows that $v_{\varepsilon} \circ \theta$ is subharmonic entirely on $\Delta$. So we may apply Fornaess-Narasimhan's criterion to conclude that $v_{\varepsilon} \in P S H^{-}(V)$. Therefore, $v_{\varepsilon} \leq v$ on $V$. By letting $\varepsilon \downarrow 0$ we obtain $v^{*} \leq v$ on $V \backslash F$. This finishes the proof since the reverse inequality is clear.

Now we turn to some basic notions of pluripotential theory on complex varieties which are involved in the statement of Theorem 1.3. Let $V$ be a complex variety of pure dimension $k$ in a bounded domain $D \subset \mathbb{C}^{n}$. According to Bedford in [Be] (see also [De] and [Ze]), the complex Monge-Ampère operator

$$
\left(d d^{c}\right)^{k}: P S H(V) \cap L_{\mathrm{loc}}^{\infty}(V) \rightarrow M_{n, n}(V),
$$

where $M_{n, n}(V)$ denotes Radon measures on $V$, may be defined in the usual way on the regular locus $V_{r}$ of $V$ (cf. [Kl] p.113), and it extends "by zero" through the singular locus $V_{s}$ i.e., for Borel sets $E \subset V$

$$
\int_{E}(d d \psi)^{k}:=\int_{E \cap V_{r}}\left(d d^{c} \psi\right)^{k}, \forall \psi \in P S H(V) \cap L_{\mathrm{loc}}^{\infty}(V) .
$$

Following Bedford and Taylor (cf. Theorem 4.4.2 in [Kl]), this operator can be used to characterize maximality of locally bounded plurisubharmonic functions on smooth complex varieties. Moreover, we may use $\left(d d^{c}\right)^{k}$ to identify pluripolar subsets of $V$. Recall that, $X \subset V$ is called pluripolar if for every $a \in X$ there exists a neighborhood $U$ of $a$ in $V$ and $v \in P S H(U)$ such that $v \not \equiv-\infty$ and $\left.v\right|_{X \cap U}=-\infty$. For instance, the singular locus $V_{S}$ of $V$ is pluripolar (in $V$ ) being a proper complex subvariety of $V$. It is well known that $\left(d d^{c} \psi\right)^{k}$ does not charge (Borel) pluripolar sets for locally bounded plurisubharmonic functions $\psi$. A major problem in pluripotential theory is to decide when a (locally) pluripolar set is globally pluripolar i.e., there exists $v \in P S H(V), v \not \equiv-\infty$ such that $\left.v\right|_{V} \equiv-\infty$. Using again the operator $\left(d d^{c}\right)^{k}$, Bedford proved that every locally pluripolar subset of $V$ is globally pluripolar provided that $V$ is Stein (cf. Theorem 5.3 in $[\mathrm{Be}]$ ). Note that in the case where $V$ is an open subset of $\mathbb{C}^{n}$, this statement is a celebrated theorem of Josefson. 
Our final ingredients consists of a few standard facts about upper semicontinuous and lower semicontinuous functions on compact sets of $\mathbb{C}^{n}$. First, we have an elementary yet useful result of Choquet (cf. Lemma 2.3.4 in [K1]).

Lemma 2.5. Let $\left\{u_{\alpha}\right\}_{\alpha \in \mathscr{A}}$ be a family of upper semicontinuous functions on $\bar{V}$ which is locally bounded from above. Then there exists a countable subfamily $\mathscr{B}$ of $\mathscr{A}$ such that

$$
\left(\sup \left\{u_{\alpha}: \alpha \in \mathscr{B}\right\}\right)^{*}=\left(\sup \left\{u_{\alpha}: \alpha \in \mathscr{A}\right\}\right)^{*} .
$$

If $u_{\alpha}$ are lower semicontinuous then $\mathscr{B}$ can be chosen so that

$$
\sup \left\{u_{\alpha}: \alpha \in \mathscr{B}\right\}=\sup \left\{u_{\alpha}: \alpha \in \mathscr{A}\right\} .
$$

The next two simple lemmas deal with sequences of upper and lower semicontinuous on compact sets of $\mathbb{C}^{n}$.

Lemma 2.6. Let $\left\{f_{j}\right\}_{j \geq 1}$ is a decreasing sequence of upper semicontinuous functions defined on a compact $K \subset \mathbb{C}^{n}$ and $g$ be a lower semicontinuous continuous function on $K$ such that

$$
\lim _{j \rightarrow \infty} f_{j}(x) \leq g(x), \forall x \in K .
$$

Then for every $\varepsilon>0$ there exists $j_{0}$ such that if $j \geq j_{0}$ then

$$
f_{j}(x)<g(x)+\varepsilon, \forall x \in K .
$$

Proof. For $j \geq 1$, we let $K_{j}:=\left\{x \in K: f_{j}(x)-g(x) \geq \varepsilon\right\}$. By the assumptions, we infer that $\left\{K_{j}\right\}_{j \geq 1}$ is a decreasing sequence of compact sets such that $\bigcap_{j \geq 1} K_{j}=\emptyset$. Thus we can find an index $j_{0} \geq 1$ such that $K_{j}=\emptyset$ for $j \geq j_{0}$. This proves our lemma.

Lemma 2.7. Let $X$ be a subset of $\mathbb{C}^{n}$ and $\left\{\varphi_{j}\right\}_{j \geq 1}$ be a sequence of lower semicontinuous functions on $X$ that increases to a lower semicontinuous function $\varphi$ on $X$. Then for every sequence $\left\{a_{j}\right\}_{j \geq 1} \subset X$ with $a_{j} \rightarrow a \in X$ we have

$$
\varphi(a) \leq \lim _{j \rightarrow \infty} \varphi_{j}\left(a_{j}\right)
$$

Proof. For $j \geq k$ we have $\varphi_{k}\left(a_{j}\right) \leq \varphi_{j}\left(a_{j}\right)$. By letting $j \rightarrow \infty$ and using lower semicontinuity of $\varphi_{k}$ at $a$ we obtain

$$
\varphi_{k}(a) \leq \lim _{j \rightarrow \infty} \varphi_{k}\left(a_{j}\right) \leq \lim _{j \rightarrow \infty} \varphi_{j}\left(a_{j}\right) .
$$

The desired conclusion follows by letting $k \rightarrow \infty$ in the right hand side.

\section{PROOFS OF THE MAIN RESUlTS}

Proof. ( of Proposition (1.2) Obviously $J_{z} \subset J_{z}^{c}, \forall z \in V$. Conversely, fix $z \in E$ and $\mu \in J_{z}^{c}$. For every $u \in P_{S H}^{-}(V)$ we choose a sequence $\left\{u_{j}\right\}_{j \geq 1} \subset P S H^{-}(V)$ that satisfy the conditions (i) and (ii). Then we have

$$
u_{j}(z) \leq \int_{V} u_{j} d \mu, \forall j \geq 1
$$

By letting $j \rightarrow \infty$ and making use of Fatou's lemma we get

$$
u(z) \leq \int_{V} u d \mu \text {. }
$$

Thus $\mu \in J_{z}$ as desired. 
Proof. (of Theorem 1.3) We will prove (a) and (b) simultaneously. Fix $u \in \operatorname{PSH}^{-}(V)$. We now follow closely the arguments in Theorem 3.1 of [DW]. Choose a sequence $\left\{\varphi_{j}\right\}_{j \geq 1} \subset \mathscr{C}(\bar{V})$ with $\varphi_{j} \downarrow u^{*}$ on $\bar{V}$. For every $j \geq 1$ we define $S \varphi_{j}$ and $S^{c} \varphi_{j}$ as follows

$$
\begin{array}{ll}
S \varphi_{j}(z):=\sup \left\{u(z): u \in P S H^{-}(V), u^{*} \leq \varphi_{j}\right. & \text { on } \bar{V}\}, z \in V, \\
S^{c} \varphi_{j}(z):=\sup \left\{u(z): u \in P S H_{c}^{-}(V), u \leq \varphi_{j}\right. & \text { on } \bar{V}\}, z \in \bar{V} .
\end{array}
$$

Then from Edwards' theorem and the assumption that $J_{z}=J_{z}^{c}$ for every $z \in V \backslash E$ we obtain

$$
S^{c} \varphi_{j}=S \varphi_{j} \text { on } V \backslash E .
$$

Since $\varphi_{j} \in \mathscr{C}(\bar{V})$, we infer that $\left(S \varphi_{j}\right)^{*} \leq \varphi_{j}$. Under the assumptions (i) and (ii), we may apply Lemma2.4 to obtain

$$
u_{j}:=S \varphi_{j}=\left(S \varphi_{j}\right)^{*} \in P S H^{-}(V \backslash F) \forall j \geq 1 .
$$

On the other hand, since $S^{c} \varphi_{j}$ is lower semicontinuous on $V$ we deduce that $\left(S \varphi_{j}\right)^{*}$ is continuous at every point in $V \backslash(E \cup F)$. Furthermore, we observe that $u \leq S \varphi_{j} \leq \varphi_{j}$ on $V$ for every $j$. Therefore $u_{j}=S \varphi_{j} \downarrow u$ on $V$. Thus we get the assertion (a) of the theorem.

Next, we let $\left\{K_{j}\right\}_{j \geq 1}$ be an exhaustion of $V^{\prime}:=V \backslash(\bar{E} \cup F)$ by compact subsets. For every $j \geq 1$, by Choquet's topological lemma2.5, we can find a sequence $\left\{v_{l, j}\right\}_{l \geq 1} \subset \operatorname{PSH}_{c}^{-}(V)$ that increases to $S^{c} \varphi_{j}$ on $\bar{V}$. By Dini's theorem and continuity of $S \varphi_{j}$ on $V^{\prime}$, the convergence is uniform on $K_{j}$ as $l \rightarrow \infty$. Thus we can choose $v_{l(j), j} \in \operatorname{PSH}_{c}^{-}(V)$ such that

$$
\left\|S \varphi_{j}-v_{l(j), j}\right\|_{K_{j}} \leq 1 / j, v_{l(j), j} \leq \varphi_{j} \text { on } \partial V .
$$

It is easy to check that $v_{j}:=v_{l(j), j}$ converges pointwise to $u$ on $V^{\prime}$ and

$$
\varlimsup_{j \rightarrow \infty} v_{j}^{*} \leq \lim _{j \rightarrow \infty} \varphi_{j}=u^{*} \text { on } \bar{V} .
$$

(c) Under the additional assumptions on $V, E$ and $u$, we will first show that the above construction of $\left\{v_{j}\right\}_{j \geq 1}$ can be modified so that $v_{j}$ is locally uniformly bounded on $V$ and that $v_{j}$ converges to $u$ locally in capacity on $V$. The desired conclusion on weak ${ }^{*}$ - convergence of Monge-Ampère measures would then follows from a result of Xing (cf. Theorem 1 in [Xi]).

Return to the proof, we let $\varphi \in P S H(V), \varphi \not \equiv-\infty$ such that $\left.\varphi\right|_{\bar{E}}=-\infty$. Let $\left\{V_{j}\right\}_{j \geq 1}$ be an exhaustion of $V$ by relatively compact open subsets. For $j \geq 1$ we define the open set $W_{j}:=\{z \in V: \varphi(z)<-j\}$. Since $u$ is bounded from below on $V_{j}$ for every $j$, we infer that the sequence $\left\{\varphi_{j}^{\prime}\right\}_{j \geq 1} \subset \mathscr{C}(\bar{V})$ defined by

$$
\varphi_{j}^{\prime}:=\max \left\{\varphi_{j}, \alpha_{j}\right\}, \text { where } \alpha_{j}=\inf _{V_{j}} u, \forall j \geq 1,
$$

decreases to $u$ on $V$ as well. Define the envelopes $S^{c} \varphi_{j}^{\prime}$ and $S \varphi_{j}^{\prime}$ as in (3.1). Then we have

$$
S^{c} \varphi_{j}^{\prime}=S \varphi_{j} \text { on } V \backslash \bar{E} .
$$

Choose a sequence $\left\{v_{l, j}\right\}_{l \geq 1} \subset P S H_{c}^{-}(V)$ that increases to $S^{c} \varphi_{j}$ on $\bar{V}$ such that $v_{l, j} \geq \alpha_{j}$ on $V_{j}$ for every $l \geq 1$. By Dini's theorem and continuity of $S \varphi_{j}$ on $V \backslash \bar{E}$ we can choose $l(j) \geq 1$ such that

$$
\left\|S \varphi_{j}^{\prime}-v_{l(j), j}\right\|_{\overline{V_{j}} \backslash W_{j}} \leq 1 / j, v_{l(j), j} \leq \varphi_{j} \text { on } \partial V .
$$

It is easy to check that the sequence $v_{j}:=v_{l(j), j}$ is locally uniformly bounded on $V$ and satisfies the conditions given in (b). Next, fix $z_{0} \in V$, we must show that there exists a small neighborhood $U$ of $z_{0}$ such that $\left(d d^{c} v_{j}\right)^{k}$ converges weakly to $\left(d d^{c} u\right)^{k}$ on $U$. Consider two cases. 
Case 1. $z_{0} \in V_{r}$. Choose a neighborhood $U$ of $z_{0}$ in $V$ which is biholomorphic to an open subset of $\mathbb{C}^{k}$. It suffices to show $\left(d d^{c} v_{j}\right)^{k}$ converges to $\left(d d^{c} u\right)^{k}$ on $U$. For simplicity of exposition, we may assume $U \subset \mathbb{C}^{k}$. Fix $\varepsilon>0$ and a relatively compact Borel subset $W$ of $U$. We claim that

$$
\lim _{j \rightarrow \infty} C\left(\left\{z \in W:\left|v_{j}(z)-u(z)\right|>\varepsilon\right\}, U\right)=0 .
$$

Recall that for a Borel subset $X$ of $U$, the relative capacity (or Bedford-Taylor capacity) $C(X, U)$ is defined as

$$
C(X, U):=\sup \left\{\int_{X}\left(d d^{c} v\right)^{k}: v \in P S H(U),-1<v<0\right\} .
$$

Using Bedford-Taylor's theorem on quasi-continuity of $u$ (cf. Theorem 3.5.5. in [Kl]), there exists a compact set $F \subset U$ such that $\left.u\right|_{F}$ is continuous whereas $C(U \backslash F, U)<\varepsilon$. Choose $j_{0}$ so large that $C\left(U \cap W_{j_{0}}, U\right)<\varepsilon$. Since $S \varphi_{j}^{\prime} \downarrow u$ on $F \backslash W_{j_{0}}$ and since $S \varphi_{j}^{\prime}$ is continuous on $F \backslash W_{j_{0}}$ for $j>j_{0}$, by Dini's theorem $S \varphi_{j}^{\prime}$ converges uniformly to $u$ on $F \backslash W_{j_{0}}$. Combining this with (3.2) we see that if $j$ is sufficiently large then

$$
\left\{z \in W:\left|v_{j}(z)-u(z)\right|>\varepsilon\right\} \subset W \cap W_{j_{0}} .
$$

The claim (3.3) follows from the choice of $j_{0}$. By applying the above mentioned theorem of Xing to $\left\{v_{j}\right\}_{j \geq 1}, u$ and the open set $U$ we conclude that $\left(d d^{c} v_{j}\right)^{k}$ converges weakly to $\left(d d^{c} u\right)^{k}$ on $U$.

Case 2. $z_{0} \in V_{s}$. Let $U$ be a neighborhood of $z_{0}$ which is relatively compact in $V$. Since $\left\{v_{j}\right\}_{j \geq 1}$ is uniformly bounded on $U$, the Chern-Levine-Nirenbeg's inequality (cf. Proposition 3.4.2 in [Kl]) implies that the measures $\left(d d^{c} v_{j}\right)^{k}$ has uniformly bounded masses on compact sets of $U \backslash V_{s}$. On the other hand, by Lemma 3.1 in [Be], the set $U \cap V_{s}$ has outer capacity zero in $U$. It follows that $\left(d d^{c} v_{j}\right)^{k}$ is uniformly bounded on compact sets of $U$. Let $\mu \in M_{n, n}(U)$ be a cluster point of the sequence $\left\{\left(d d^{c} v_{j}\right)^{k}\right\}_{j \geq 1}$ in the weak * topology. By the forgoing case, we know already that $\mu=\left(d d^{c} u\right)^{k}$ on $V_{r} \cap U$. Moreover, the vanishing of the outer capacity of $U \cap V_{s}$ also yields that $\mu=\left(d d^{c} u\right)^{k}=0$ on $V_{s} \cap U$. Therefore $\mu=\left(d d^{c} u\right)^{k}$ on $U$. Thus $\left(d d^{c} v_{j}\right)^{k}$ converges to $\left(d d^{c} u\right)^{k}$ on $U$ in the weak ${ }^{*}$ - topology.

The proof is thereby completed.

Proof. (of Theorem 1.4) (a) Obviously, $J_{z} \subset J_{z}^{c}, \forall z \in V$. So it is enough to show the reverse conclusion. Fix $z_{0} \in V \backslash E$, a measure $\mu \in J_{z_{0}}^{c}$ and $u \in P S H^{-}(V)$. Since $V$ is Stein, by Fornaess-Narasimhan's approximation theorem there exist sequences $\left\{u_{j}\right\}_{j \geq 1},\left\{v_{j}\right\}_{j \geq 1} \subset$ $P S H(V) \cap \mathscr{C}(V)$ with $u_{j} \downarrow u$ and $v_{j} \downarrow v$ on $V$. Moreover, by the upper semicontinuity of $u$ on $\bar{V}$ we can find a sequence $\left\{\varphi_{j}\right\}_{j \geq 1} \subset \mathscr{C}(\bar{V})$ such that $\varphi_{j}<0, \varphi_{j} \downarrow u$ on $\bar{V}$. For each $j \geq 1$, we define the upper envelope

$$
S^{c} \varphi_{j}(z)=\sup \left\{u: u \in P S H^{c}(V), u \leq \varphi_{j} \text { on } \bar{V}\right\}, z \in \bar{V} .
$$

Then

$$
S^{c} \varphi_{j} \leq \varphi_{j} \text { on } \bar{V}
$$

By Edward's theorem 2.2 and the hypothesis (ii) we obtain

$$
S^{c} \varphi_{j}=\varphi_{j} \text { on }(\partial V) \backslash K .
$$

By Choquet's topological lemma2.5, there exists an increasing sequence $\left\{\varphi_{j, k}\right\}_{k \geq 1} \subset P S H^{c}(V)$ such that

$$
\varphi_{j, k} \uparrow S^{c} \varphi_{j} \text { on } \bar{V} \text { as } k \rightarrow \infty \text {. }
$$


Let $\left\{V_{j}\right\}_{j \geq 1}$ be an exhaustion of $V$ by relatively compact open subsets. Fix $\varepsilon>0$ and integers $j \geq 1, p \geq 1$, we claim that there exist an index $k(j)>j$ such that for every $l \geq k(j)$ we have

$$
\varphi_{j, l} \geq u+\varepsilon v-\frac{\varepsilon}{2} \text { on } \partial V_{l} \text {. }
$$

Indeed, suppose that (3.7) is false. Then there exists a sequence $\left\{z_{k_{l}}\right\}_{l \geq 1} \subset V$ with $z_{k_{l}} \in \partial V_{k_{l}}$ such that

$$
\varphi_{j, k_{l}}\left(z_{k_{l}}\right)<u\left(z_{k_{l}}\right)+\varepsilon v\left(z_{k_{l}}\right)-\frac{\varepsilon}{2} .
$$

By passing to a subsequence if necessary, we can assume that $z_{k_{l}} \rightarrow z^{*} \in \partial V$ as $l \rightarrow \infty$. In view of Lemma 2.7 and the upper semicontinuity of $u$ we get

$$
S^{c} \varphi_{j}\left(z^{*}\right) \leq u\left(z^{*}\right)+\varepsilon v^{*}\left(z^{*}\right)-\frac{\varepsilon}{2} .
$$

Note that the left hand side of (3.8) is bounded from below by $\inf _{\partial V} \varphi_{j}>-\infty$, so $z^{*} \notin K$ by the assumption (i). Then it follows from (3.5) and (3.8), that

$$
\varphi_{j}\left(z^{*}\right) \leq u\left(z^{*}\right)+\varepsilon v\left(z^{*}\right)-\frac{\varepsilon}{2}<u\left(z^{*}\right) .
$$

This contradicts the fact that $\varphi_{j} \downarrow u$. Hence the claim (3.7) is proved.

Now, from (3.7) and Lemma 2.6 we infer that for every $l \geq k(j)$ there exists an index $m(l) \geq$ $k(j)$ such that

Using Lemma2.3 we see that the function

$$
\varphi_{j, l} \geq u_{m(l)}+\varepsilon v_{m(l)}-\varepsilon \text { on } \partial V_{l} .
$$

$$
\tilde{u}_{j, p, l}:= \begin{cases}\max \left\{\varphi_{j, l}, u_{m(l)}+\varepsilon v_{m(l)}-\varepsilon\right\} & \text { on } V_{l} \\ \varphi_{j, l} & \text { on } \bar{V} \backslash V_{l}\end{cases}
$$

belongs to $\mathrm{PSH}^{c}(V)$. Furthermore, it follows from (3.6) that

$$
\tilde{u}_{j, p, l} \rightarrow \theta_{j, p}:= \begin{cases}\max \left\{S^{c} \varphi_{j}, u+\varepsilon v-\varepsilon\right\} & \text { on } V \\ S^{c} \varphi_{j} & \text { on } \partial V\end{cases}
$$

as $l \rightarrow \infty$. In particular, since $v<0$ on $V$, by (3.4) we conclude that

$$
u+\varepsilon v-\varepsilon \leq \theta_{j, p} \text { on } V, \theta_{j, p} \leq \varphi_{j} \text { on } \bar{V} \text {. }
$$

Using $\mu \in J_{z_{0}}^{c}$ we obtain

$$
\tilde{u}_{i, p . l}\left(z_{0}\right) \leq \int_{\bar{V}} \tilde{u}_{j, p, l} d \mu=\int_{V} \tilde{u}_{j, p, l} d \mu+\int_{\partial V} \tilde{u}_{j, p, l} d \mu .
$$

By letting $l \rightarrow \infty$, using Fatou's lemma, by (3.9) and noting that $v<0$ on $V$ we obtain the following estimates

$$
u\left(z_{0}\right)+\varepsilon v\left(z_{0}\right)-\varepsilon \leq \theta_{j, \varepsilon}\left(z_{0}\right) \leq \int_{\bar{V}} \varphi_{j} d \mu .
$$

Letting $j \rightarrow \infty$ and then $\varepsilon \rightarrow 0$ (taking into account that $v\left(z_{0}\right)>-\infty$ ) applying Fatou's lemma again we get

$$
u\left(z_{0}\right) \leq \int_{\bar{V}} u d \mu
$$

This means that $\mu \in J_{z_{0}}$, we are done.

(b) Let $\left\{\varphi_{j}\right\}_{j \geq 1} \subset \mathscr{C}(\bar{V})$ be the sequence chosen in (a). For each $j \geq 1$ we define the envelope

$$
S \varphi_{j}(z)=\sup \left\{u(z): u \in P S H^{-}(V), u \leq \varphi_{j} \text { on } \bar{V}\right\}, z \in V .
$$


It follows from (a) and the proof of Theorem 1.3 (a) that $u_{j}:=S \varphi_{j} \in P S H^{-}(V)$ and the sequence $u_{j} \downarrow u$ on $V \backslash E$ as $j \rightarrow \infty$. Moreover, $u_{j}$ is continuous at every point of $V \backslash E$. For boundary behavior of $u_{j}$ we fix $\xi \in(\partial V) \backslash K$. Then we have

$$
\varphi_{j}(\xi)=S^{c} \varphi_{j}(\xi) \leq \varliminf_{z \rightarrow \xi, z \in V} S^{c} \varphi_{j}(z) \leq \varliminf_{z \rightarrow \xi, z \in V} u_{j}(z) .
$$

Therefore $\lim _{z \rightarrow \xi} u_{j}(z)=\varphi_{j}(\xi)$. Thus $u_{j}$ is continuous at every point of $U:=\bar{V} \backslash(K \cup E)$ and $u_{j}^{*} \downarrow u^{*}$ on $U$. The proof is complete.

For the proof of the next theorem we require the following fact.

Lemma 3.1. Let $V$ be a complex variety in a bounded domain $D \subset \mathbb{C}^{n}$ and $\xi \in \partial V$ be a boundary point. Assume that there is a local continuous plurisubharmonic barrier at $\xi$. Then for every sequence $\left\{\varphi_{j}\right\}_{j \geq 1} \subset \mathscr{C}(\partial V), \varphi_{j}<0$ that decreases to an upper semicontinuous function $\varphi$ on $\partial V$ and every sequence $\left\{\xi_{j}\right\} \subset V$ with $\xi_{j} \rightarrow \xi$ we have

$$
\varlimsup_{j \rightarrow \infty} S^{c} \varphi_{j}\left(\xi_{j}\right) \leq \varphi(\xi),
$$

where $S^{c} \varphi_{j}(z):=\sup \left\{u(z): u \in \operatorname{PSH}_{c}^{-}(V), u \leq \varphi_{j}\right.$ on $\left.\partial V\right\}, z \in \bar{V}$.

Proof. Let $u$ be a local continuous plurisubharmonic barrier at $\xi$. By the argument given in the remark following Theorem 1.4, we may extend $u$ to $\tilde{u} \in P S H_{c}^{-}(V)$ such that $\tilde{u}$ is a barrier at $\xi$. Let $\left\{\mu_{j}\right\}_{j \geq 1}, \mu_{j} \in J_{z_{j}}$ be a sequence of Jensen measures with compact support in $\partial V$. We claim that $\mu_{j}$ converges to $\delta_{\xi}$ in the weak ${ }^{*}$ - topology. It suffices to show that any cluster point of this sequence coincides with $\delta_{\xi}$. Let $\mu^{*}$ be such a cluster point. Then we have

$$
0=\varliminf_{j \rightarrow \infty} \tilde{u}\left(z_{j}\right) \leq \varliminf_{j \rightarrow \infty} \int_{V} \tilde{u} d \mu_{j} \leq \int_{V} \tilde{u} d \mu^{*} \leq 0 .
$$

So $\mu^{*}=\delta_{\xi}$. The proves the claim. It follows, since $\varphi_{j} \downarrow \varphi$ on $\partial V$, that

$$
\varlimsup_{j \rightarrow \infty} S^{c} \varphi_{j}\left(z_{j}\right) \leq \varlimsup_{j \rightarrow \infty} \int_{\partial V} \varphi_{j} d \mu_{j} \leq \lim _{j \rightarrow \infty} \int_{\partial V} \varphi_{j} d \delta_{\xi}=\varphi(\xi) .
$$

This is the desired conclusion.

Proof. (of Theorem 1.5) We split the proof in two two parts.

Existence. After subtracting a large constant we may assume $\varphi<0$ on $\partial V$. Define the upper envelopes

$$
\begin{aligned}
& S \varphi(z):=\sup \left\{u(z): u \in P S H^{-}(V), u \leq \varphi \quad \text { on } \partial V\right\}, z \in V \\
& S^{c} \varphi(z):=\sup \left\{u(z): u \in P S H_{c}^{-}(V), u^{*} \leq \varphi \quad \text { on } \partial V\right\}, z \in \bar{V} .
\end{aligned}
$$

In view of the assumption (a) and the remark following Theorem 1.4 we have $J_{\xi}^{c}=\left\{\delta_{\xi}\right\}$ for every $\xi \in(\partial V) \backslash K$. So by Edwards' duality theorem (with $\varphi:=+\infty$ on $V$ ) we obtain

$$
S^{c} \varphi=\varphi \text { on }(\partial V) \backslash K \text {. }
$$

Furthermore, since $V$ is Stein, by Theorem 1.4 (a) we get $J_{z}=J_{z}^{c}$ for every $z \in V$. So using again Edwards' duality theorem as above we get

$$
S \varphi=S^{c} \varphi \text { on } V .
$$

Since $V$ is locally irreducible, $u:=\left(S^{c} \varphi\right)^{*} \in P S H^{-}(V)$. Moreover, by the assumption (ii) we may apply Lemma 3.1 to $\varphi_{j}=\varphi$ to obtain

$$
\varlimsup_{z \rightarrow \xi, z \in V} u(z) \leq \varphi(\xi), \forall \xi \in(\partial V) \backslash K .
$$


Fix $\varepsilon>0$ and set $u_{\varepsilon}:=u+\varepsilon v$. Then we infer from the last inequality and the assumption that $\left(u_{\varepsilon}\right)^{*} \leq \varphi$ on $\partial V$. This implies that $u_{\varepsilon} \leq S \varphi$ on $V$. By letting $\varepsilon \downarrow 0$ and noting that $v>-\infty$ on $V$ we get $u=S \varphi=S^{c} \varphi$ on $V$. Hence $u$ is lower semicontinuous on $V$, so $u \in P S H(V) \cap \mathscr{C}(V)$ and $\|u\|_{V} \leq\|\varphi\|_{\partial V}$. Next we show that $u$ has the right boundary values. Indeed, fix $\xi \in(\partial V) \backslash K$ and a sequence $\xi_{j} \rightarrow \xi, \xi_{j} \in V$. By lower semicontinuity on $\bar{V}$ of $S^{c} \varphi$ and (3.11), (3.13) we have

$$
\varphi(\xi)=S^{c} \varphi(\xi) \leq \varliminf_{j \rightarrow \infty} u\left(\xi_{j}\right) \leq \varlimsup_{j \rightarrow \infty} u\left(\xi_{j}\right) \leq \varphi(\xi) .
$$

It follows that $u(z) \rightarrow \varphi(\xi)$ as $z \rightarrow \xi, z \in V$. Finally, we let $w \in P S H(V)$ with $w \leq u$ on $V \backslash U$ for some open set $U$ relatively compact in $V$. Then by the gluing lemma 2.3

$$
\tilde{u}(z):= \begin{cases}\max \{u(z), w(z)\}, & z \in U \\ u(z) & z \in V \backslash U\end{cases}
$$

belongs to $P S H(V)$. Moreover, $\tilde{u}$ is a member in the defining family for $S \varphi$. Therefore $\tilde{u} \leq$ $S \varphi=u$ on $V$. In particular, $w \leq u$ on $U$. This proves maximality of $u$ and also completes the proof of the existence of the solution.

Uniqueness. Assume that there exist bounded continuous plurisubharmonic functions $u_{1}, u_{2}$ on $V$ such that

$$
\lim _{z \rightarrow \xi, z \in V} u_{1}(z)=\lim _{z \rightarrow \xi, z \in V} u_{2}(z)=v(\xi), \forall \xi \in(\partial V) \backslash K
$$

Let $\left\{V_{j}\right\}$ be a sequence of relative compact open subset of $V$ with $V_{j} \uparrow V$. Fix $\varepsilon>0$, since $u_{2}$ is bounded from below on $V$ we can find $j_{0} \geq 1$ so large such that

$$
u_{1}+\varepsilon v \leq u_{2}+\varepsilon \text { on } V \backslash V_{j_{0}} .
$$

It follows from maximality of $u_{2}$ that

$$
u_{1}+\varepsilon v \leq u_{2}+\varepsilon \text { on } V .
$$

By letting $\varepsilon \downarrow 0$, we infer that $u_{1} \leq u_{2}$ on $V$. Similarly we also get $u_{2} \leq u_{1}$ on $V$. Therefore $u_{1}=u_{2}$ on $V$.

The theorem is proved.

Proof. (of Therem (1.6) For each $j \geq 1$, put

$$
V_{j}:=\left\{z \in V: \rho(z)<-\frac{1}{2 j^{2}}\right\} .
$$

Then $V_{j}$ is a relative compact open subset of $V, \forall j \geq 1$ and $V_{j} \uparrow V$. By the upper semicontinuity of $u^{*}$ on $\bar{V}$ there exists a sequence $\varphi_{j} \in \mathscr{C}(\bar{V}), \varphi_{j}<0$ such that $\varphi_{j} \downarrow u^{*}$ on $\bar{V}$. FornaessNarasimhan's approximation theorem yields a sequence $\left\{v_{j}\right\}_{j \geq 1} \subset P S H(V) \cap \mathscr{C}(V)$ such that $v_{j} \downarrow u$ on $V$. Now for $j \geq 1$ we define the upper envelopes

$$
\begin{array}{ll}
S \varphi_{j}(z):=\sup \left\{u(z): u \in P S H^{-}(V), u^{*} \leq \varphi_{j}\right. & \text { on } \bar{V}\}, z \in V \\
S^{c} \varphi_{j}(z):=\sup \left\{u(z): u \in P S H_{c}^{-}(V), u \leq \varphi_{j}\right. & \text { on } \bar{V}\}, z \in \bar{V} .
\end{array}
$$

Using Edwards' duality theorem and the assumption (i) we obtain

$$
S^{c} \varphi_{j}=\varphi_{j} \text { on } \partial V \text {. }
$$

Furthermore, by Theorem 1.4 (a) we get $J_{z}=J_{z}^{c}$ for every $z \in V$. So by applying again Edwards' duality theorem as above we get

$$
u \leq S \varphi_{j}=S^{c} \varphi_{j} \text { on } V .
$$


Next, fix $j \geq 1$, in view of Choquet's topological lemma 2.5 there is a sequence $\left\{\varphi_{j, l}\right\}_{l \geq 1} \subset$ $\mathrm{PSH}_{c}^{-}(V)$ that increases to $S^{c} \varphi_{j}$ on $\bar{V}$. So, using (3.14), (3.15) and Lemma 2.6 we can find $k(j)$ such that

$$
u \leq \varphi_{j, k(j)}+\frac{1}{3 j} \text { on } \overline{V_{j}}
$$

and

$$
\max \left\{u^{*}, \varphi_{j}-\frac{1}{j}\right\} \leq \varphi_{j, k(j)} \leq \varphi_{j} \text { on } \partial V .
$$

Hence, it follows from Lemma 2.6 and (3.16) that there exists $l(j) \geq j$ satisfying

$$
v_{l(j)} \leq \varphi_{j, k(j)}+\frac{1}{2 j} \text { on } \bar{V}_{j}
$$

Now we consider the new function

$$
\tilde{u}_{j}:= \begin{cases}\max \left\{j \rho+\varphi_{j, k(j)}, v_{l(j)}-\frac{1}{j}\right\} & \text { on } V_{j} \\ j \rho+\varphi_{j, k(j)} & \text { on } \bar{V} \backslash V_{j} .\end{cases}
$$

Observe that, on $\partial V_{j}$ we have

$$
v_{l(j)}-\frac{1}{j}=\left(v_{l(j)}-\frac{1}{2 j}\right)-\frac{1}{2 j} \leq \varphi_{j, k(j)}-\frac{1}{2 j}=j \rho+\varphi_{j, k(j)} .
$$

Therefore, by the gluing lemma $2.3 \tilde{u}_{j} \in P S H^{c}(V)$ and $\left.\tilde{u}_{j}\right|_{\partial V}=\varphi_{j, k(j)}$. This implies that $\tilde{u}_{j} \rightarrow$ $u^{*}$ pointwise on $\bar{V}$ as $j \rightarrow \infty$. Furthermore, since $\rho<0$ on $V$ the following estimates hold on $\bar{V}$

$$
\tilde{u}_{j} \leq \varphi_{j, k(j)}+\frac{1}{j} .
$$

Fix $j \geq 1$, for each $p \geq j$ we set

$$
h_{p, j}:=\sup _{j \leq m \leq p} \tilde{u}_{m} \text { and } u_{j}=\sup _{j \leq m} \tilde{u}_{m} .
$$

It is then clear that $h_{p, j} \in P S H^{c}(V)$ and $h_{p, j} \uparrow u_{j}$ on $\bar{V}$. Moreover, by the choice of $k(j)$ we have

$$
\varphi_{j}-\frac{1}{j} \leq \varphi_{j, k(j)} \leq u_{j} \leq \varphi_{j} \text { on } \partial V .
$$

This implies that $u_{j} \downarrow u^{*}$ on $\bar{V}$. We claim that $h_{p, j}$ is uniformly convergent to $u_{j}$ on $\bar{V}$ as $p \rightarrow \infty$. Assume otherwise, there exist $\varepsilon>0$ and a sequence $\left\{z_{p}\right\} \subset \bar{V}$ satisfying

$$
h_{p, j}\left(z_{p}\right)+\varepsilon<u_{j}\left(z_{p}\right), \forall p \geq j .
$$

Since $u_{j}$ is lower semicontinuous on $\bar{V}$, we may assume $\left\{z_{p}\right\}_{p \geq 1} \subset V$. By the definition of $u_{j}$ and since 3.19, there exists $m(p)>p$ such that

$$
h_{p, j}\left(z_{p}\right)+\frac{\varepsilon}{2}<\tilde{u}_{m(p)}\left(z_{p}\right), \forall p \geq j .
$$

After switching to a subsequence if necessary, we can assume that $z_{p} \rightarrow z^{*} \in \bar{V}$. We consider two cases.

Case 1. $z^{*} \in V$. Then we may find $p_{0} \geq 1$ such that $\left\{z_{p}\right\}_{p \geq 1} \cup\left\{z^{*}\right\} \subset V_{m\left(p_{0}\right)}$. By the definition of $\tilde{u}_{j}$ and taking into account the fact that $\rho<0$ on $V$ we obtain

$$
\tilde{u}_{m(p)}=v_{l(m(p))}-\frac{1}{m(p)} \text { on } V_{m(p)}
$$

for all $p \geq p_{0}$ large enough. This implies that

$$
\varlimsup_{p \rightarrow \infty} \tilde{u}_{m(p)}\left(z_{p}\right)=\varlimsup_{p \rightarrow \infty} v_{l(m(p))}\left(z_{p}\right) \leq \varlimsup_{p \rightarrow \infty} v_{p}\left(z_{p}\right) \leq u\left(z^{*}\right),
$$


where the last inequality follows from Lemma 2.7. Moreover, the left hand side of (3.20) is bounded from below by $\inf _{\bar{V}} \tilde{u}_{j}+\frac{\varepsilon}{2}>-\infty$, therefore $u\left(z^{*}\right)>-\infty$.

On the other hand, using Lemma 2.7 again we obtain

$$
\varliminf_{p \rightarrow \infty} h_{p, j}\left(z_{p}\right) \geq u_{j}\left(z^{*}\right) \geq \varliminf_{p \rightarrow \infty} \tilde{u}_{m(p)}\left(z^{*}\right)=\varliminf_{p \rightarrow \infty}\left(v_{l(m(p))}\left(z^{*}\right)-\frac{1}{m(p)}\right)=u\left(z^{*}\right) .
$$

Thus, by letting $p \rightarrow \infty$ in (3.20) and taking limsup in both sides we get a contradiction.

Case 2. $z^{*} \in \partial V$. It follows from (3.20) and (3.18) that

$$
\begin{aligned}
\tilde{u}_{j}\left(z_{p}\right)+\frac{\varepsilon}{2} & \leq h_{p, j}\left(z_{p}\right)+\frac{\varepsilon}{2}<\tilde{u}_{m(p)}\left(z_{p}\right) \leq \varphi_{m(p), k\left(m_{p}\right)}\left(z_{p}\right)+\frac{1}{m(p)} \\
& \leq S^{c} \varphi_{m(p)}\left(z_{p}\right)+\frac{1}{m(p)} \leq \varphi_{m(p)}\left(z_{p}\right)+\frac{1}{m(p)} \leq \varphi_{p}\left(z_{p}\right)+\frac{1}{m(p)}, \forall p \geq j .
\end{aligned}
$$

By taking limsup in both sides as $p \rightarrow \infty$ and noting that $u_{j}=\varphi_{j, k(j)}$ on $\partial V$ we obtain

$$
\varphi_{j, k(j)}\left(z^{*}\right)+\frac{\varepsilon}{2}=\tilde{u}_{j}\left(z^{*}\right)+\frac{\varepsilon}{2} \leq \varlimsup_{p \rightarrow \infty} \varphi_{p}\left(z_{p}\right) \leq u^{*}\left(z^{*}\right) .
$$

Here the last inequality follows from Lemma 2.7 and the fact that $\varphi_{p} \downarrow u^{*}$ on $\bar{V}$. This is a contradiction to (3.17).

Thus, our claim is fully proved. Since $h_{p, j} \in P S H^{c}(V)$, it follows from Fornaess-Narasimhan's criterion that $u_{j} \in \mathrm{PSH}_{c}^{-}(V)$. The proof is complete.

\section{References}

[Be] E. Bedford, The operator $\left(d d^{c}\right)^{n}$ on complex spaces, Séminaire d'Analyse Lelong-Skoda, Lecture Notes in Math., 919 (1981), 294-324.

[Ch] E. M. Chirka, Complex Analytic Sets, Kluwer, Dordecht, 1989.

[De] J.-P. Demailly, Mesures de Monge-Ampère et caractérisation géométrique des variétés algébriques affines, Mém. Soc. Math. France (N.S.) 19 (1985), 1-124.

[Ed] D. A. Edwards, Choquet boundary theory for certain spaces of lower semicontinuous functions, in Function Algebras (Proc. Internat. Symposium on Function Algebras, Tulane Univ., 1965) (Scott-Foresman, Chicago, 1966), pp. 300-309.

[DW] N.Q. Dieu and F. Wikström, Jensen measures and approximation of plurisubharmonic functions, Michigan Math. J. 53 (2005) 529-544.

[Di] Nguyen Quang Dieu, Approximation of plurisubharmonic functions on bounded domains in $\mathbb{C}^{n}$, Michigan Math. J. 54, (2006) 697-711.

[FN] J. E. Fornaess and R. Narasimhan, The Levi problem on complex spaces with singularities, Math. Ann. 248 (1980) 47-72.

[Kl] M. Klimek, Pluripotential Theory, Oxford 1991.

[Si] N. Sibony, Une classe de domaines pseudoconvexes, Duke Math. J. 55 (1987), 299-319.

[Wik1] F. Wikström, Jensen measures and boundary values of plurisubharmonic functions, Ark. Mat. 39 (2001) 181-200.

[Wik2] F. Wikström, The Dirichlet problem for maximal plurisubharmonic functions on analytic varieties in $\mathbb{C}^{n}$, International Journal of Mathematics 20, No. 4 (2009) 521-528. 
[Ze] A. Zeriahi, Fonction de Green pluricomplexe à pole à l'infini sur un espace de Stein parabolique et applications, Math. Scand. 69 (1991), 89-126.

Department of Mathematics, Hanoi National University of Education, 136 Xuan Thuy StreEt, CAU Giay, HaNoi, Vietnam

E-mail address: ngquangdieu@hn.vnn.vn, tangvan.long@gmail.com, sanphetMA@gmail.com 